\title{
Matching an oscillator model to a phase response curve
}

\author{
Pierre Sacré and Rodolphe Sepulchre
}

\begin{abstract}
The Phase Response Curve (PRC) has proven a useful tool for the reduction of complex oscillator models. It is also an information often experimentally available to the biologist. This paper introduces a numerical tool based on the sensitivity analysis of the PRC to adapt initial model parameters in order to match a particular PRC shape. We illustrate the approach on a simple biochemical model of circadian oscillator.
\end{abstract}

\section{INTRODUCTION}

Rhythmic phenomena are essential to the dynamic behavior of biological systems [1]-[3]. They arise in genetic and metabolic networks as a result of complex interactions between multiple biological processes, which makes their design principles not intuitive. Elucidating those underlying mechanisms is crucial to advances in systems biology.

Quantitative models based firmly on experiments provide an essential tool for studying those mechanisms. With recent advances in biology, the number of identified key variables in a given process increases and the nature of their interactions (feedforward and feedback loops) is better known. In spatially homogeneous conditions, ordinary differential equations describe the time evolution of the system; yet current models suffer from several limitations. Among others, parameter values are often determined empirically or based on the few pieces of experimental information.

In mathematical biology, the Phase Response Curve (PRC) has proven a useful input-output tool for the reduction of complex oscillator models [4]-[6]. It indicates how the timing of inputs affects the timing (steady-state phase-shift) of oscillators. Not surprisingly, the shape of this curve plays a critical role in entrainment and synchronization properties of the system [7], [8]. Moreover, the PRC is well adapted to description tools developed by biologists. It can often be experimentally measured for circadian rhythms [9], [10].

We developed a numerical tool to adapt an initial choice of parameters in order to match an oscillator model to a particular PRC. We propose a natural distance between equivalence classes of PRCs and perform a gradient-descent search in the model parameter space. The gradient computation involves the sensitivity of the PRC.

The paper is organized as follows. Section II introduces the notion of PRC in the neighborhood of a stable periodic orbit. Section III provides the sensitivity analysis of a periodic orbit and its PRC. Section IV defines a metric between equivalent PRCs and derives its associated gradient in the parameter space. Section V applies a gradient-descent algorithm based on this distance to the Goodwin oscillator model.

P. Sacré and R. Sepulchre are with the Department of Electrical Engineering and Computer Science (B28), University of Liège, 4000 Liège, Belgium (e-mail: pierre.sacre@ulg.ac.be, r.sepulchre@ulg.ac.be).

\section{DERIVING A PHASE RESPONSE CURVE}

IN THE NEIGHBORHOOD OF A STABLE PERIODIC ORBIT

In this section, we introduce basic definitions about periodic orbits and summarize the phase reduction procedure leading to the notion of PRC. Details about this standard procedure can be found in [4]-[6].

\section{A. Closed system and hyperbolic stable periodic orbit}

We consider a closed dynamical system defined by a set of first-order ordinary differential equations

$$
\dot{x}=f(x, p), \quad x(0)=x^{0},
$$

in which states $x(t)$ evolve on some subset $\mathcal{X} \subseteq \mathbb{R}^{n}$ and (constant) parameters $p$ take values in some subset $\mathcal{P} \subseteq \mathbb{R}^{q}$. The vector field $f: \mathcal{X} \times \mathcal{P} \rightarrow \mathbb{R}^{n}$ supports all the usual smoothness conditions that are necessary for existence and uniqueness of solutions. The flow (arising from the vector field $f$ ) is the map $\phi$ that associates to $\left(t, x^{0}, p\right) \in \mathbb{R} \times \mathcal{X} \times \mathcal{P}$ the solution $\phi\left(t, x^{0}, p\right)=x(t)$ at time $t \in \mathbb{R}$. The orbit of the flow $\phi$ through $x^{0} \in \mathcal{X}$ for fixed parameters $p \in \mathcal{P}$ is the invariant set $\Gamma:=\left\{x \in \mathcal{X}: x=\phi\left(t, x^{0}, p\right), t \in \mathbb{R}\right\}$.

For fixed parameters $p$, we assume that the system (1) admits a periodic orbit $\gamma \subset \mathcal{X}$ with period $T$ (and corresponding angular frequency $\omega=2 \pi / T)$. The periodic orbit $\gamma$ is the invariant set described by a (non-constant) $T$-periodic solution $\phi\left(t, x_{\gamma}^{0}, p\right)=x_{\gamma}(t)$ which is defined for all times $t \in \mathbb{R}$ and where $x_{\gamma}^{0}$ is a reference position on the periodic orbit $\gamma$. The period $T$ is the smallest positive number with the property that $\phi\left(T, x_{\gamma}^{0}, p\right)=x_{\gamma}^{0}$.

As the reference position $x_{\gamma}^{0}$ may be any point on the periodic orbit $\gamma$, there are infinitely many solutions describing the same periodic orbit $\gamma$. A unique isolated solution can be selected by imposing a phase condition

$$
\varphi\left(x_{\gamma}^{0}, T, p\right)=0
$$

where $\varphi: \gamma \times \mathbb{R}_{>0} \times \mathcal{P} \rightarrow \mathbb{R}$ is a smooth map. Examples of valid phase conditions can be found in [11].

The periodic solution $x_{\gamma}(\cdot)$ with the period $T$ can be computed by solving the boundary value problem (BVP) as

$$
\begin{aligned}
\dot{x}_{\gamma}(t)-f\left(x_{\gamma}(t), p\right) & =0 \\
x_{\gamma}(T)-x_{\gamma}(0) & =0 \\
\varphi\left(x_{\gamma}^{0}, T, p\right) & =0 .
\end{aligned}
$$

This periodic BVP is a particular case of a two-point BVP.

In what follows, we assume that the periodic orbit $\gamma$ possesses two crucial properties: asymptotic orbital stability and asymptotic phase property (see [12] for definitions). 
Assuming hyperbolic stability of the periodic orbit provides those properties as stated by the Andronov-Witt theorem.

Theorem 1 (Andronov-Witt theorem [12]): If 1 is a simple characteristic multiplier of the variational system of (1) and the remaining $n-1$ characteristic multipliers are in modulus less than one (i.e. if the periodic orbit is hyperbolically stable), then the periodic solution $x_{\gamma}(t)$ is asymptotically orbitally stable, having the asymptotic phase property.

The maximal open set from which the periodic orbit $\gamma$ attracts is called the basin (or the oscillator stable set) of $\gamma$

$$
\mathcal{B}(\gamma):=\left\{x^{0} \in \mathcal{X}: \lim _{t \rightarrow+\infty} \operatorname{dist}\left(\phi\left(t, x^{0}, p\right), \gamma\right)=0\right\}
$$

where

$$
\operatorname{dist}(x, \gamma):=\inf _{y \in \gamma}\|x-y\|_{2}
$$

is the distance from the point $x \in \mathcal{X}$ to the set $\gamma \subset \mathcal{X}$ based on the Euclidean norm $\|\cdot\|_{2}$ in $\mathbb{R}^{n}$.

\section{B. Phase, asymptotic phase, and isochron}

Any point $x^{0} \in \gamma$ can be characterized by a scalar phase $\vartheta^{0} \in \mathbb{S}^{1}$ that uniquely determines the position of the point $x^{0}$ along the periodic orbit $\gamma$ because a periodic orbit is a onedimensional (closed) curve homeomorphic to $\mathbb{S}^{1}$. For fixed parameters $p$, the smooth bijective phase map $\Theta: \gamma \rightarrow \mathbb{S}^{1}$ associates to each point $x^{0}$ on the periodic orbit its phase $\vartheta^{0}$, such that

$$
\left\|\phi\left(t, x^{0}, p\right)-x_{\gamma}\left(t+\vartheta^{0} / \omega\right)\right\|_{2}=0, \quad \text { for all } t \in \mathbb{R} .
$$

This mapping is constructed such that the image of $x_{\gamma}^{0}$ is equal to 0 and the progression along $\gamma$ (in absence of perturbation) produces a constant increase in $\vartheta^{0}$. The phase variable $\vartheta: \mathbb{R}_{\geq 0} \rightarrow \mathbb{S}^{1}$ is defined for each trajectory $\phi\left(t, x^{0}, p\right)$ starting from a point $x^{0}$ on the periodic orbit $\gamma$, as $\vartheta(t)=\Theta\left(\phi\left(t, x^{0}, p\right)\right)=\Theta\left(x_{\gamma}\left(t+\vartheta^{0} / \omega\right)\right)$. The phase variable $\vartheta$ evolves linearly in time

$$
\dot{\vartheta}(t)=\omega, \quad \vartheta(0)=\vartheta^{0},
$$

such that $\vartheta(t)=\omega t+\vartheta_{0} \bmod 2 \pi$.

For a periodic orbit with the asymptotic phase property, the notion of phase can be extended to any point $x^{0}$ in the basin $\mathcal{B}(\gamma)$ by defining the concept of asymptotic phase.For fixed parameters $p$, the asymptotic phase map $\Theta: \mathcal{B}(\gamma) \rightarrow \mathbb{S}^{1}$ associates to each point $x^{0}$ in the basin $\mathcal{B}(\gamma)$ its asymptotic phase $\theta^{0}$, such that

$$
\lim _{t \rightarrow+\infty}\left\|\phi\left(t, x^{0}, p\right)-x_{\gamma}\left(t+\theta^{0} / \omega\right)\right\|_{2}=0 .
$$

Again, the mapping is constructed such that the image of $x_{\gamma}^{0}$ is equal to 0 and the progression along any orbit $\Gamma$ (in absence of perturbation) produces a constant increase in $\theta^{0}$. The asymptotic phase variable $\theta: \mathbb{R}_{\geq 0} \rightarrow \mathbb{S}^{1}$ is defined along each solution $\phi\left(t, x^{0}, p\right)$ starting in the basin of attraction of $\gamma$, as $\theta(t)=\Theta\left(\phi\left(t, x^{0}, p\right)\right)$. The asymptotic phase variable $\theta$ follows

$$
\dot{\theta}(t)=\omega, \quad \theta(0)=\theta^{0},
$$

such that $\theta(t)=\omega t+\theta^{0} \bmod 2 \pi$.
The set of all points having the same asymptotic phase is called an isochron. Considering hyperbolic periodic orbit, isochrons are codimension-1 submanifolds (diffeomorphic to $\mathbb{R}^{n-1}$ ) crossing the periodic orbit transversally [13].

\section{Open system and (input) infinitesimal PRC}

The dynamical system in (1) is turned into an open system

$$
\dot{x}=f(x, p)+\epsilon g(x, p) u(t), \quad x\left(t^{0}\right)=x^{0},
$$

where input values $u(t)$ belong to some subset $\mathcal{U} \in \mathbb{R}^{m}$. The map $g: \mathcal{X} \times \mathcal{P} \rightarrow \mathbb{R}^{n \times m}$ supports all the usual conditions that are necessary for existence and uniqueness of solutions. The parameter $\epsilon$ is assumed to be small $(0<\epsilon \ll 1)$ in order to weakly perturb the closed system. The flow arising from this open system is the map $\phi_{u}$ that associates to $\left(t, t^{0}, x^{0}, u, p\right) \in \mathbb{R} \times \mathbb{R} \times \mathcal{X} \times \mathcal{U} \times \mathcal{P}$ the solution $\phi_{u}\left(t, t^{0}, x^{0}, u, p\right)$ at time $t \in \mathbb{R}$.

The phase variable corresponding to a solution of the open system (3) is defined as $\theta(t)=\Theta\left(\phi_{u}\left(t, t^{0}, x^{0}, u, p\right)\right)$. Using the chain rule, we have

$$
\frac{d \Theta}{d t}\left(\phi_{u}\right)=\omega+\epsilon \nabla_{x} \Theta^{T}\left(\phi_{u}\right) g\left(\phi_{u}, p\right) u
$$

(where we omitted the argument $\left(t, t^{0}, x^{0}, u, p\right)$ for notational convenience). Evaluating the right side of this equation at the intersection of the isochron corresponding to $\phi_{u}$ and the periodic orbit $\gamma$,

$$
x_{\gamma}\left(\Theta\left(\phi_{u}\right) / \omega\right)=\left\{x \in \gamma: \Theta(x)=\Theta\left(\phi_{u}\right)\right\},
$$

the evolution of the phase variable is approximated by a onedimensional nonlinear equation

$$
\dot{\theta} \approx \omega+\epsilon \nabla_{x} \Theta^{T}\left(x_{\gamma}(\theta / \omega)\right) g\left(x_{\gamma}(\theta / \omega), p\right) u(t)
$$

subject to the initial condition $\theta(0)=\Theta\left(x^{0}\right)$. This equation is valid (up to the first-order approximation) in a neighborhood of the periodic orbit $\gamma$.

The gradient map $\nabla_{x} \Theta: \mathcal{B}(\gamma) \rightarrow \mathbb{R}^{n}$ measures the relative infinitesimal asymptotic phase-shift caused by an infinitesimal state perturbation. When evaluated along the periodic orbit, the map $\nabla_{x} \Theta\left(\phi\left(\tau, x_{\gamma}^{0}, p\right)\right)=q(t)$ is known as the (state) infinitesimal Phase Response Curve (iPRC).

The directional derivative $L_{g} \Theta: \mathcal{B}(\gamma) \rightarrow \mathbb{R}^{m}$ is defined as

$$
L_{g \cdot i} \Theta(x)=g_{\cdot i}^{T}(x, p) \nabla_{x} \Theta(x)
$$

in which $g_{\cdot i}$ is the $i$ th column of the matrix $g$. When evaluated along the periodic orbit, the map $L_{g} \Theta\left(\phi\left(\tau, x_{\gamma}^{0}, p\right)\right)=$ $q_{u}(t)$ is known as the (input) iPRC. It serves as a $\delta$-impulse response characteristics in the direction of phase-shift.

The iPRC $q(\cdot)$ is the $T$-periodic solution of the adjoint variational system (Malkin theorem, see [4] or [6])

$$
\begin{aligned}
-\dot{q}-f_{x}^{*}\left(x_{\gamma}(t), p\right) q & =0 \\
q(T)-q(0) & =0 \\
\left\langle f\left(x_{\gamma}(t), p\right), q(t)\right\rangle & =\omega
\end{aligned}
$$

where $f_{x}$ denotes the Jacobian matrix of $f$ with respect to $x$. This normalization condition (4c) ensures that the asymptotic phase variable of the closed system evolves linearly in time. 


\section{Sensitivity Analysis of}

A PERIODIC ORBIT AND ITS PHASE RESPONSE CURVE

In this section, we introduce the sensitivity analysis of a periodic orbit and its iPRC. Sensitivity analysis of iPRCs was previously applied in the context of electronics in [14].

\section{A. Classical sensitivity analysis of a (periodic) solution}

Classical sensitivity analysis considers the effects at time $t$ on a state value $x_{i}$ of perturbing (at time 0 ) a parameter $p_{j}$

$$
s_{i j}^{x}(t):=\frac{d \phi_{i}}{d p_{j}}\left(t, x^{0}, p\right) .
$$

From the differential equation

$$
\frac{d \phi}{d t}\left(t, x^{0}, p\right)=f\left(\phi\left(t, x^{0}, p\right), p\right)
$$

we have, taking derivatives with respect to $p$,

$$
\frac{d}{d t}\left(\frac{d \phi}{d p}\right)=\frac{d}{d p}\left(\frac{d \phi}{d t}\right)=f_{x}(\phi, p)\left(\frac{d \phi}{d p}\right)+f_{p}(\phi, p)
$$

(where $f_{p}$ denotes the Jacobian matrix of $f$ with respect to $p$ and we omitted the argument $\left(t, x^{0}, p\right)$ in order to not clutter the formula). In addition, from the identity $\phi\left(0, x^{0}, p\right)=x^{0}$, we also have that

$$
\frac{d \phi}{d p}\left(0, x^{0}, p\right)=\frac{d x^{0}}{d p} .
$$

Therefore the sensitivity of a solution $\frac{d \phi}{d p}\left(t, x^{0}, p\right)=S^{x}(t)$ is the solution of the matrix equation

$$
\dot{S}^{x}(t)=f_{x}\left(\phi\left(t, x^{0}, p\right), p\right) S^{x}(t)+f_{p}\left(\phi\left(t, x^{0}, p\right), p\right),
$$

subject to the initial conditions $S^{x}(0)=\frac{d x^{0}}{d p}$.

In the analysis of oscillatory systems, we focus on the $T$-periodic solution $\phi\left(t, x_{\gamma}^{0}, p\right)$. The general solution of (5) about a periodic solution takes the form (see [15]-[17])

$$
S_{\gamma}^{x}(t)=t R_{\gamma}^{x}(t)+Z_{\gamma}^{x}(t)
$$

The first term $t R_{\gamma}^{x}(t)$ is unbounded. It contains the influence of the period sensitivity $\frac{d T}{d p}$ (row vector in $\mathbb{R}^{q}$ ) on the sensitivity of the solution through the $T$-periodic matrix

$$
R_{\gamma}^{x}(t)=-\frac{1}{T} f\left(\phi\left(t, x_{\gamma}^{0}, p\right), p\right) \frac{d T}{d p} .
$$

The second term $Z_{\gamma}^{x}(t)$ is bounded and $T$-periodic. It represents the sensitivity of the periodic orbit. From now, we will drop the index $\gamma$ to simplify notations.

\section{B. Sensitivity of the periodic orbit}

In order to focus on the sensitivity of the periodic orbit, we introduce the normalized time $s=t / T$ and the corresponding flow $\tilde{\phi}\left(s, x^{0}, p\right):=\phi\left(T s, x^{0}, p\right)$. The sensitivity of the periodic orbit is then defined as

$$
z_{i j}^{x}(t):=\frac{d \tilde{\phi}_{i}}{d p_{j}}\left(s, x_{\gamma}^{0}, p\right) .
$$

From the differential equation (2a) written for the normalized time

$$
\frac{d \tilde{\phi}}{d s}\left(s, x_{\gamma}^{0}, p\right)-T f\left(\tilde{\phi}\left(s, x_{\gamma}^{0}, p\right), p\right)=0,
$$

we have, taking derivatives with respect to $p$,

$$
\frac{d}{d s} \frac{d \tilde{\phi}}{d p}-T f_{x}(\tilde{\phi}, p) \frac{d \tilde{\phi}}{d p}-T f_{p}(\tilde{\phi}, p)-f(\tilde{\phi}, p) \frac{d T}{d p}=0
$$

(where we omitted the argument $\left(s, x_{\gamma}^{0}, p\right)$ in order to not clutter the formula). In addition, from the phase condition $(2 \mathrm{c})$, we have

$$
\frac{\partial \varphi}{\partial x^{0}} \frac{d x_{\gamma}^{0}}{d p}+\frac{\partial \varphi}{\partial T} \frac{d T}{d p}+\frac{\partial \varphi}{\partial p}=0
$$

(where we omitted the argument $\left(x_{\gamma}^{0}, T, p\right)$ in order to not clutter the formula).

The sensitivity of the periodic orbit $Z^{x}(t)$ and the period sensitivity $\frac{d T}{d p}$ are solutions of the BVP

$$
\begin{aligned}
\dot{Z}^{x}(t)-f_{x}\left(x_{\gamma}(t), p\right) Z^{x}(t)-\frac{1}{T} \dot{x}_{\gamma}(t) \frac{d T}{d p} & =B^{x}(t) \\
Z^{x}(T)-Z^{x}(0) & =0 \\
\frac{\partial \varphi}{\partial x^{0}} Z^{x}(0)+\frac{\partial \varphi}{\partial T} \frac{d T}{d p} & =-\frac{\partial \varphi}{\partial p}
\end{aligned}
$$

with $B^{x}(t)=f_{p}\left(x_{\gamma}(t), p\right)$.

\section{Sensitivity of the infinitesimal phase response curve}

Similarly to the sensitivity of the periodic orbit, we define the sensitivity of the iPRC as

$$
z_{i j}^{q}(t):=\frac{d \nabla_{x_{i}} \Theta}{d p_{j}}\left(\tilde{\phi}\left(s, x_{\gamma}^{0}, p\right)\right) .
$$

From the differential equation (4a) written for the normalized time

$$
-\frac{d \nabla_{x} \Theta}{d s}(\tilde{\phi})-T f_{x}^{*}(\tilde{\phi}, p) \nabla_{x} \Theta(\tilde{\phi})=0
$$

we have, taking derivatives with respect to $p$,

$$
-\frac{d}{d s} \frac{d \nabla_{x} \Theta}{d p}(\tilde{\phi})-T f_{x}^{*}(\tilde{\phi}, p) \frac{d \nabla_{x} \Theta}{d p}(\tilde{\phi})=T \tilde{B}^{q}(s)
$$

in which elements $\tilde{b}_{i j}^{q}(s)$ of the matrix $\tilde{B}^{q}(s) \in \mathbb{R}^{n \times p}$ are given by

$$
\sum_{k=1}^{n}\left(\frac{1}{T} \frac{\partial \bar{f}_{k}}{\partial x_{i}} \frac{d T}{d p_{j}}+\sum_{l=1}^{n} \frac{\partial^{2} \bar{f}_{k}}{\partial x_{i} \partial x_{l}} \frac{d \tilde{\phi}_{l}}{d p_{j}}+\frac{\partial^{2} \bar{f}_{k}}{\partial x_{i} \partial p_{j}}\right) \nabla_{x_{k}} \Theta
$$

(where we omitted all arguments in order to not clutter the formula). In addition, from the normalization condition (4c), we have

$$
f^{T}(\tilde{\phi}, p) \frac{d \nabla_{x} \Theta}{d p}(\tilde{\phi})+\nabla_{x} \Theta^{T}(\tilde{\phi}) Z^{f}(t)=\frac{d \omega}{d p}
$$

with $Z^{f}(t)=f_{x}(\tilde{\phi}, p) Z^{x}(t)+f_{p}(\tilde{\phi}, p)$.

The sensitivity of the iPRC $Z^{q}(\cdot)$ is the unique $T$-periodic solution of the BVP

$$
\begin{aligned}
-\dot{Z}^{q}(t)-f_{x}^{*}\left(x_{\gamma}(t), p\right) Z^{q}(t) & =B^{q}(t) \\
Z^{q}(T)-Z^{q}(0) & =0 \\
f^{T}\left(x_{\gamma}(t), p\right) Z^{q}(t)+q^{T}(t) Z^{f}(t) & =\frac{d \omega}{d p}
\end{aligned}
$$

with $B^{q}(t)=\tilde{B}^{q}(t / T)$. 


\section{ShAPE-DISTANCE METRIC AND GRADIENT}

We aim at selecting an oscillator model by adapting initial parameters to better match a given PRC. Discriminating between points in the parameter space requires to quantify the similarity between an oscillator PRC and a reference signal (both possibly depending on parameters $p$ ). To our knowledge, an empirical similarity measure between PRCs was only used once in the context of oscillator model reduction [18].

In this section, we construct a shape-distance metric between equivalent signals and derive the associated gradient in the parameter space.

\section{A. Shape-distance between equivalent periodic signals}

We consider two periodic signals $x(\cdot) \in L_{2}\left[0, T_{x}\right)$ and $y(\cdot) \in L_{2}\left[0, T_{y}\right)$ with different periods being the oscillator PRC (for parameters $p$ ) and a reference signal, respectively. To compare one cycle of $x(\cdot)$ to one cycle of $y(\cdot)$, a timescaling transformation is applied to both signals. Time-scaled signals $\tilde{x}(t)=x\left(\frac{T_{x}}{T} t\right)$ and $\tilde{y}(t)=\left(\frac{T_{y}}{T} t\right)$ are $T$-periodic and belong to the same vector space $L_{2}[0, T)$. This vector space is equipped with the scalar product

$$
\langle\tilde{x}(\cdot), \tilde{y}(\cdot)\rangle=\int_{0}^{T} \tilde{x}(t) \overline{\tilde{y}}(t) d t
$$

where $\overline{\tilde{y}}(t)$ denotes the complex conjugate of $\tilde{y}(t)$. The scalar product induces the associated norm

$$
\|\tilde{x}(\cdot)\|_{2}=\langle\tilde{x}(\cdot), \tilde{x}(\cdot)\rangle^{1 / 2}=\left(\int_{0}^{T}|\tilde{x}(t)|^{2} d t\right)^{1 / 2} .
$$

The distance induced by the norm in this vector space is

$$
d(\tilde{x}(\cdot), \tilde{y}(\cdot))=\|\tilde{x}(\cdot)-\tilde{y}(\cdot)\|_{2} .
$$

We show in the following how to modify this natural distance to satisfy two equivalence properties, namely timeshifting and magnitude-scaling equivalence properties. From now, we will intentionally omit the symbols $\sim$ in order to not clutter the notations.

Signals are considered as equivalent up to a time-shifting and a magnitude-scaling if they are related as follow

$$
x(\cdot) \sim y(\cdot) \Leftrightarrow \exists \sigma \in[0, T), \alpha \in \mathbb{R}_{>0}: y(\cdot)=x(\cdot-\sigma) / \alpha .
$$

The set of all signals equivalent to $x(\cdot)$ is the equivalence class $[x(\cdot)]$.

We define the distance between two equivalence classes as

$$
d_{c}([x(\cdot)],[y(\cdot)]):=\min _{\xi \in[x], \chi \in[y]} \frac{d(\xi(\cdot), \chi(\cdot))}{\sqrt{\|\xi(\cdot)\|_{2}\|\chi(\cdot)\|_{2}}}
$$

in which $\|\xi(\cdot)\|_{2} \neq 0$ and $\|\chi(\cdot)\|_{2} \neq 0$ if we consider nontrivial signals. The normalization is necessary in order to avoid all distances to tend towards zero.

This minimization problem can be reformulated as

$$
\min _{\sigma \in[0, T), \alpha \in \mathbb{R}_{>0}} \frac{d(x(\cdot), \alpha y(\cdot+\sigma))}{\sqrt{\alpha\|x(\cdot)\|_{2}\|y(\cdot)\|_{2}}}
$$

and then, using the definition of the induced distance, as

$$
\min _{\sigma \in[0, T) \alpha \in \mathbb{R}_{>0}} \sqrt{\frac{1}{\alpha} \frac{\|x(\cdot)\|_{2}}{\|y(\cdot)\|_{2}}+\alpha \frac{\|y(\cdot)\|_{2}}{\|x(\cdot)\|_{2}}-2 \Re e\left\{\rho_{x y}(\sigma)\right\}}
$$

in which $\rho_{x y}(\sigma)$ is a short notation for the normalized circular cross-correlation

$\rho(x(\cdot), y(\cdot), \sigma)=\frac{\overline{\langle x(\cdot), y(\cdot+\sigma)\rangle}}{\|x(\cdot)\|_{2}\|y(\cdot)\|_{2}}=\int_{0}^{T} \frac{\bar{x}(t) y(t+\sigma)}{\|x(\cdot)\|_{2}\|y(\cdot)\|_{2}} d t$.

The circular cross-correlation $\rho_{x y}(\cdot)$ is a $T$-periodic signal which measures the similarity between two signals as a function of the time-lag $\sigma$ applied to one of them. Minimizations over $\sigma$ and $\alpha$ can be done separately and optimal solutions $\left(\sigma_{*}, \alpha_{*}\right)$ are given by

$$
\begin{aligned}
& \sigma_{*}(x(\cdot), y(\cdot))=\arg \max _{\sigma \in[0, T)} \Re e\{\rho(x(\cdot), y(\cdot), \sigma)\}, \\
& \alpha_{*}(x(\cdot), y(\cdot))=\frac{\|x(\cdot)\|_{2}}{\|y(\cdot)\|_{2}} .
\end{aligned}
$$

The distance between equivalence classes of periodic signals is thus eventually given by

$$
d_{c}([x(\cdot)],[y(\cdot)])=\sqrt{2\left(1-\Re e\left\{\rho\left(x(\cdot), y(\cdot), \sigma_{*}\right)\right\}\right)}
$$

with $\sigma_{*}(x(\cdot), y(\cdot))=\arg \max _{\sigma \in[0, T)} \Re e\{\rho(x(\cdot), y(\cdot), \sigma)\}$.

\section{B. Shape-distance gradient}

To explore the parameter space with a gradient-descent algorithm, we need the gradient $\nabla_{p} d_{c}(\cdot, \cdot)$ defined by the distance $d_{c}(\cdot, \cdot)$ between equivalence classes of periodic signals $x(\cdot, p)$ and $y(\cdot, p)$. Both signals may depend on parameters $p$. The circular cross-correlation $\rho\left(x(\cdot, p), y(\cdot, p), \sigma_{*}\right)$ involved in the distance expression depends directly on signals $x(\cdot, p)$ and $y(\cdot, p)$ but also indirectly through the timelag $\sigma_{*}(x(\cdot, p), y(\cdot, p))$.

Using the chain rule, we have

$$
\Re e\left\{\frac{d \rho}{d p}\right\}=\Re e\left\{\frac{\partial \rho}{\partial x} \frac{d x}{d p}+\frac{\partial \rho}{\partial y} \frac{d y}{d p}+\frac{\partial \rho}{\partial \sigma} \frac{d \sigma_{*}}{d p}\right\}
$$

(where we omitted arguments in order to not clutter the formula). The last term is equal to zero because we have

$$
\Re e\left\{\frac{\partial \rho}{\partial \sigma}\left(x(\cdot, p), y(\cdot, p), \sigma_{*}\right)\right\}=0
$$

by definition of $\sigma_{*}$. First and second terms are computed easily by straightforward derivatives.

The shape-distance gradient is thus eventually given by

$$
\nabla_{p} d_{c}([x(\cdot, p)],[y(\cdot, p)])=-\frac{\Re e\left\{\frac{\partial \rho}{\partial x} \frac{d x}{d p}+\frac{\partial \rho}{\partial y} \frac{d y}{d p}\right\}}{\sqrt{2\left(1-\Re e\left\{\rho\left(x, y, \sigma_{*}\right)\right\}\right)}} .
$$

\section{Gradient-descent algorithm}

We use a gradient-descent algorithm with Armijo step sizes for line search. Its descent direction at each iteration is the opposite of the gradient and the Armijo step size gives an approximate line minimization [19].

In general, we are dealing with non-convex optimization problems (non-convex cost functions over non-convex sets). The gradient-descent algorithm will reach a local minimum which may not be the global minimum of the problem. 


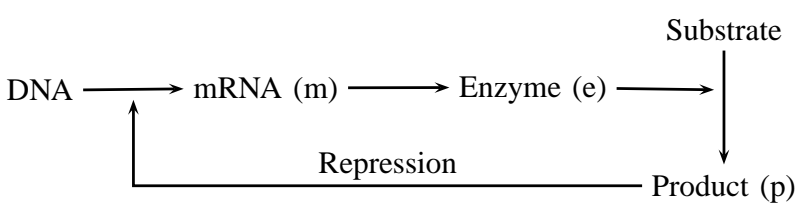

(a)

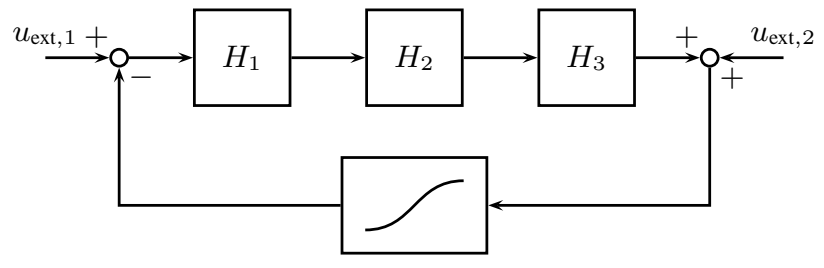

(b)

Fig. 1. Goodwin oscillator. (a) Biological interpretation. (b) Input-output scheme representing the mathematical model.

\section{Application to Goodwin oscillator}

In this section, we apply our gradient-descent algorithm to a simple biochemical model of circadian oscillator.

\section{A. Goodwin oscillator}

The Goodwin oscillator is a cyclic feedback system where metabolites repress the enzymes which are essential for their own synthesis by inhibiting the transcription of the molecule DNA to messenger RNA (mRNA) [20]. The model for such a mechanism is schematically shown in Fig. 1. It can be described as the cyclic interconnection of three first-order subsystems and a monotone static nonlinearity

$$
\begin{aligned}
& H_{i}:\left\{\begin{array}{r}
\tau_{i} \dot{x}_{i}=K_{i} u_{i}-x_{i} \\
y_{i}=x_{i}
\end{array} \quad \text { for } \quad i=1, \ldots, 3\right. \\
& H_{4}:\left\{y_{4}=-\frac{1}{1+\left(u_{4} / \theta\right)^{p}}\right.
\end{aligned}
$$

where the cyclic interconnection is given by $u_{1}=u_{\text {ext, } 1}-y_{4}$, $u_{2}=y_{1}, u_{3}=y_{2}$, and $u_{4}=u_{\text {ext }, 2}+y_{3}$. Two external input channels represent two ways to perturb the biological oscillator. A dimensionless form of this system is equivalent to impose $K_{2}=K_{3}=\tau_{1}=\theta=1$.

This system possesses a single equilibrium in $x_{1}=x_{2}=$ $x_{3}=x^{*}$, with $x^{*}$ being the solution of $\frac{K_{1}}{1+x^{p}}-x=0$. The equilibrium may loose its stability only through a Hopf bifurcation giving birth to a stable periodic orbit [21], [22].

To simplify the analysis (but without loss of generality), we reduce the parameter space to two dimensions: we impose equal time-constants in $H_{2}$ and $H_{3}\left(\tau_{2}=\tau_{3}=\tau\right)$ and fixe the Hill coefficient $p=20$. This high coefficient is justified by the necessity to get periodic orbits $(p>8)$ and strong enough differences between iPRC shapes in the parameter space. The results for weaker coefficient are similar but less marked. The parameter space reduces to $\left(K_{1}, \tau\right) \in \mathbb{R}_{>0}^{2}$.

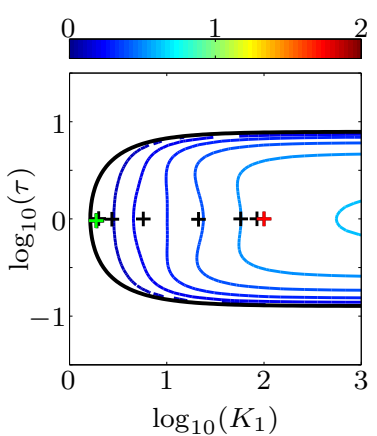

(a) First input channel.

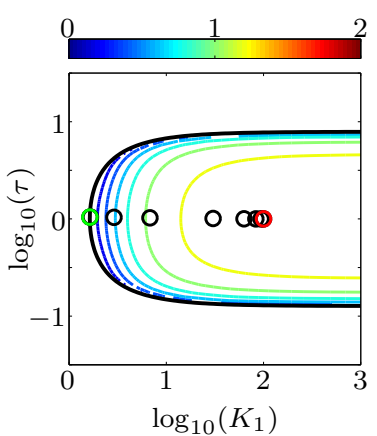

(b) Second input channel.

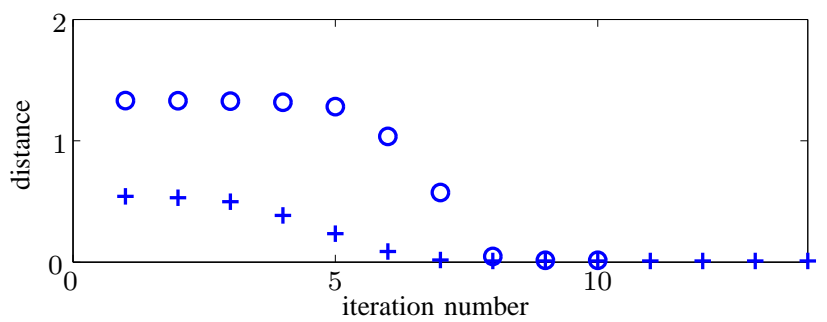

(c)

Fig. 2. Distance of iPRC for Goodwin oscillator to a Hopf-bifurcationlike iPRC. (a)-(b) The shape-distance (color levels) between a sinusoid and the (input) iPRCs corresponding to the first and second input channel increases with the distance from the Hopf-bifurcation manifold (black line). The gradient-descent algorithm follows the path indicated by + and $\circ$. (c) The distance along the path followed by the gradient-descent algorithm decreases with the iteration number.

\section{B. Minimal shape-distance to a Hopf-bifurcation-like iPRC}

Approximation of iPRCs have been computed in the neighborhood of codimension-1 bifurcations [5]. In particular, it has been shown that iPRCs are sinusoidal near Hopf bifurcations. However, the shape of those iPRCs may be modified when the system evolves away from the bifurcation.

Figures 2(a) and 2(b) show the contour levels (in the reduced parameter space) of the shape-distance between a sinusoid and (input) iPRCs corresponding, respectively, to the first and the second input channel. In both cases, the shape-distance increases (almost concentrically) with the distance to the Hopf-bifurcation manifold (black line). The shape-distance for the second input channel takes higher values indicating a larger deformation from a sinusoid.

To enlighten this observation, we apply our gradientdescent based on the shape-distance to a sinusoid. Starting from the center of the parameter space, the gradient-descent algorithm follows paths in direction of the Hopf-bifurcation manifold (Fig. 2(a) and 2(b)). The shape-distance along those paths decreases as the iteration number increases (Fig. 2(c)).

\section{Minimal shape-distance to an experimental-like iPRC}

Experimental PRCs have been measured for circadian rhythms of various organisms [9], [10]. We apply our gradient-descent algorithm to the Goodwin oscillator in order to match an experimental-like iPRC of circadian rhythms.

Figures 3(a) and 3(b) show the contour levels of the shapedistance to an experimental-like iPRC and the paths followed 


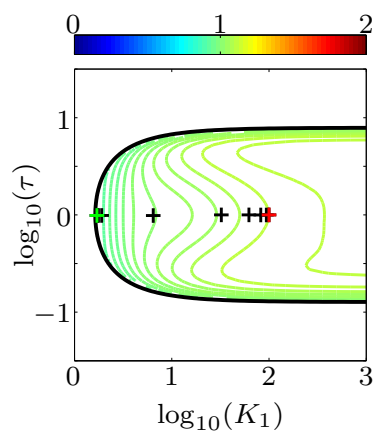

(a) First input channel.

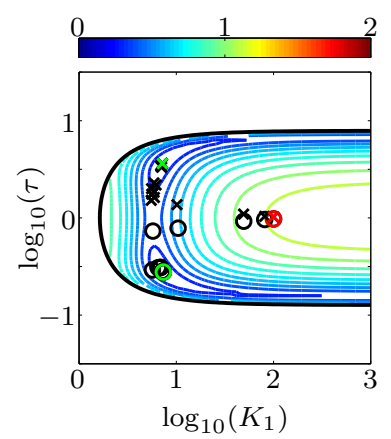

(b) Second input channel.

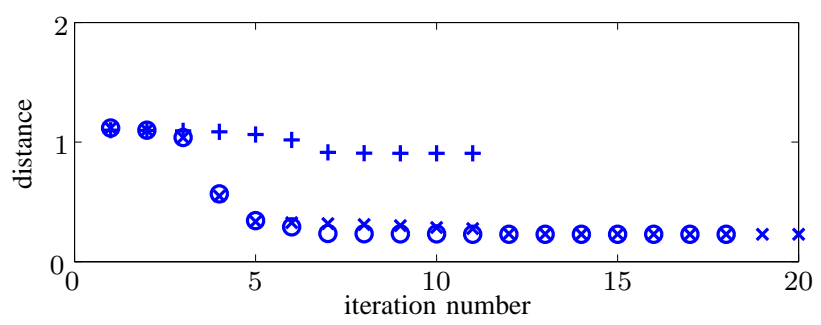

(c)

Fig. 3. Distance of iPRCs for Goodwin oscillator to an experimental-like iPRC. (a)-(b) The shape-distance (color levels) between an experimental iPRC and the (input) iPRCs corresponding to the first and second input channel exhibits a non-convex behavior in the reduced parameter space. The gradient-descent algorithm follows the path indicated by,$+ \circ$ and $\times$. (c) The distance along the path followed by the gradient-descent algorithm decreases with the iteration number.

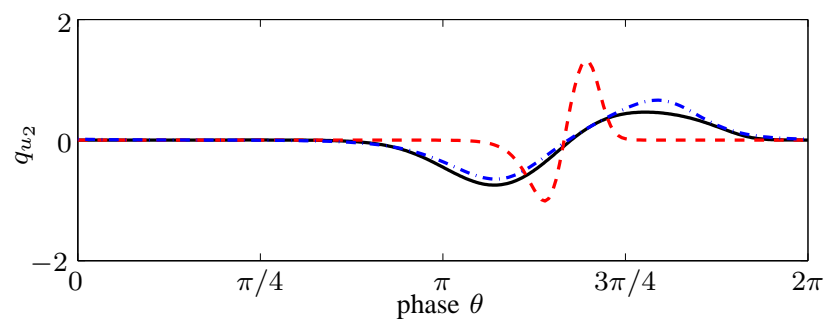

Fig. 4. The shape of the optimal iPRC (dashed-dotted line) is closer to the reference iPRC (solid line) than the initial iPRC (dashed line).

by the gradient-descent algorithm in the reduced parameter space. Two paths are shown for iPRCs corresponding to the second input channel. Starting from close initial points, paths evolve towards different local minima. The shapedistance being almost symmetric with respect to a unitary time-constant $\tau$, both local minima correspond to similar iPRCs (up to a time-shifting and a magnitude scaling).

Figure 4 compares initial (dashed) and optimal (dasheddotted) iPRCs for the second input channel to the reference one (solid). The optimal iPRC fits very well the experimental-like one.

\section{Conclusion}

We have presented a gradient-descent algorithm that allows to adapt initial parameters to reach a particular iPRC shape. This tool is based on the sensitivity analysis of the
iPRC and on a shape-distance metric between equivalence classes of periodic signals. The application of this simple tool on the Goodwin model gives encouraging results. We plan to apply those tools to more complex models of circadian rhythms [23].

\section{ACKNOWLEDGMENTS}

This paper presents research results of the Belgian Network DYSCO (Dynamical Systems, Control, and Optimization), funded by the Interuniversity Attraction Poles Programme, initiated by the Belgian State, Science Policy Office. The scientific responsibility rests with its authors. P. Sacré is supported as an F.R.S.-FNRS researcher fellow (Belgian Fund for Scientific Research).

\section{REFERENCES}

[1] A. Goldbeter, Biochemical Oscillations and Cellular Rhythms: The Molecular Bases of Periodic and Chaotic Behaviour. Cambridge University Press, 1996.

[2] A. T. Winfree, The Geometry of Biological Time. Springer, 2001.

[3] Y. Kuramoto, Chemical Oscillations, Waves, and Turbulence. Dover Publications, 2003.

[4] F. C. Hoppensteadt and E. M. Izhikevich, Weakly connected neural networks. Springer, 1997.

[5] E. Brown, J. Moehlis, and P. Holmes, "On the phase reduction and response dynamics of neural oscillator populations," Neural Comput., vol. 16, no. 4, pp. 673-715, 2004.

[6] E. M. Izhikevich, Dynamical systems in neuroscience: the geometry of excitability and bursting. The MIT Press, 2007.

[7] N. Bagheri, S. R. Taylor, K. Meeker, L. R. Petzold, and F. J. Doyle III, "Synchrony and entrainment properties of robust circadian oscillators." J. R. Soc. Interface, vol. 5 Suppl 1, pp. S17-S28, 2008.

[8] N. Bagheri, J. Stelling, and F. J. Doyle III, "Circadian phase resetting via single and multiple control targets." PLoS Comput. Biol., vol. 4, no. 7, p. e1000104, 2008.

[9] J. C. Hall and M. Rosbash, "Genes and biological rhythms," Trends in Genetics, vol. 3, pp. 185-191, 1987.

[10] C. H. Johnson, An Atlas of Phase Responses Curves for Circadian and Circatidal Rhythym. Vanderbilt University, 1990.

[11] R. Seydel, Practical Bifurcation and Stability Analysis. Springer, 2010.

[12] M. Farkas, Periodic Motions. Springer, 1994.

[13] J. Guckenheimer, "Isochrons and phaseless sets," J. Math. Biol., vol. 1, no. 3, pp. 259-273, 1975.

[14] I. Vytyaz, D. Lee, P. Hanumolu, U.-K. Moon, and K. Mayaram, "Sensitivity Analysis for Oscillators," IEEE Trans. Comput.-Aided Design Integr. Circuits Syst., vol. 27, no. 9, pp. 1521-1534, 2008.

[15] E. Rosenwasser and R. Yusupov, Sensitivity of automatic control systems. CRC Press, 2000.

[16] B. P. Ingalls, "Autonomously oscillating biochemical systems: parametric sensitivity of extrema and period," Syst. Biol., vol. 1, no. 1, pp. 62-70, 2004.

[17] A. K. Wilkins, B. Tidor, J. White, and P. I. Barton, "Sensitivity analysis for oscillating dynamical systems," SIAM J. Sci. Comput., vol. 31, no. 4, pp. 2706-2732, 2009.

[18] S. R. Taylor, F. J. Doyle III, and L. R. Petzold, "Oscillator model reduction preserving the phase response: application to the circadian clock.” Biophys. J., vol. 95, no. 4, pp. 1658-1673, Aug. 2008.

[19] J. Nocedal and S. Wright, Numerical Optimization. Springer, 2006.

[20] B. C. Goodwin, "Oscillatory behavior in enzymatic control processes." Adv. Enzyme Regul., vol. 3, pp. 425-438, 1965.

[21] J. J. Tyson and H. G. Othmer, "The dynamics of feedback control circuits in biochemical pathways," Prog. Theor. Biol., vol. 5, pp. 162, 1978.

[22] M. Arcak and E. D. Sontag, "Diagonal stability of a class of cyclic systems and its connection with the secant criterion," Automatica, vol. 42, no. 9, pp. 1531-1537, 2006.

[23] J.-C. Leloup and A. Goldbeter, "Modeling the molecular regulatory mechanism of circadian rhythms in Drosophila." BioEssays, vol. 22, no. 1, pp. 84-93, Jan. 2000. 